\title{
Impact of cold ischemia on cytokines after partial liver transplantation in rats
}

\author{
Q.-A. Qi, Z.-Y. Yang, K.-S. Ma, Q. Lu, S.-G. Wang, X.-W. Li, F. Xia, \\ W. Liu and P. Bie \\ Southwest Hospital \& Institute of Hepatobilitary Surgery, \\ Third Military Medical University, Chongqing, China \\ Corresponding author: P. Bie \\ E-mail: qiqinganbp@yeah.net
}

Genet. Mol. Res. 12 (3): 4003-4008 (2013)

Received March 2, 2013

Accepted July 29, 2013

Published September 27, 2013

DOI http://dx.doi.org/10.4238/2013.September.27.1

\begin{abstract}
To study the impact of cold ischemia on tumor necrosis factor-alpha (TNF- $\alpha$ ) and interleukin-10 (IL-10) expression after liver transplantation, a stable model of partial liver transplantation in rats was established. The experimental animals were divided into the following groups: a partial hepatectomy control group, a group that received partial liver transplantation after $30 \mathrm{~min}$ of cold ischemia (experimental group A), and a group that received a partial liver transplantation after $10 \mathrm{~h}$ of cold ischemia (experimental group B). The survival rate was observed in each group. The liver tissue was sampled 1, 2, and 4 days after surgery, and immunohistochemical detection of proliferating cell nuclear antigen TNF- $\alpha$ and IL-10 was performed. The correlation between liver regeneration and TNF- $\alpha$ and IL-10 expression was analyzed, and the impact of the 2 cytokines on rat liver regeneration after liver transplantation was evaluated. The survival rates of rats in the partial hepatectomy control group, in the group that received a partial liver transplantation after 30 min of cold ischemia, and the group that received a partial liver transplantation after $10 \mathrm{~h}$ of cold ischemia were 100,70 , and $33.3 \%$, respectively. The expression of proliferating cell nuclear antigen and TNF- $\alpha$ was decreased $(\mathrm{P}<0.05)$, and IL-10
\end{abstract}


expression was increased $(\mathrm{P}<0.05)$ in animals that received a partial liver transplant after $10 \mathrm{~h}$ of cold ischemia compared with that in the animals that received a partial liver transplant after $30 \mathrm{~min}$ of cold ischemia. We conclude that with the extension of cold ischemic time, liver regeneration and survival rate after liver transplantation decreased. TNF- $\alpha$ and IL-10 play important regulatory roles in the regeneration process of transplanted livers.

Key words: Liver transplantation; Liver regeneration; Interleukin-10; Tumor necrosis factor

\section{INTRODUCTION}

The lack of donor livers is one of the challenges in improving clinical liver transplantation (Lo, 2012). Split liver transplantation and partial living liver transplantation have opened up new avenues for solving the lack of donors, but the regenerative capacity of the liver plays an important role in the survival of the recipient after transplantation. Studies have confirmed that patients who receive partial living liver transplantation display significantly greater liver regenerative capacity compared with that of split liver transplantation recipients (Hegab et al., 2012), and this difference is due to the shorter cold ischemia time (Li et al., 2011). Recent studies have established a stable partial liver transplantation model in rats through in vivo liver resection, which removes approximately $50 \%$ of the liver, including the left lateral lobe, the partial middle lobe, and the caudate lobe (Omura et al., 1996). This model can be used to study the impact of various cold ischemia times on cytokine production and liver regeneration. Proliferating cell nuclear antigen (PCNA) is an auxiliary protein of DNA polymerase, and its synthesis is directly related to DNA replication and cell proliferation (Yu et al., 2012). Changes in PCNA expression reflect DNA synthesis and DNA replication; thus, PCNA expression can be used to determine the level of DNA synthesis in live cells and cell proliferation.

Studies have shown that the regeneration of transplanted livers is delayed after partial liver transplantation compared with that after partial hepatectomy (Kele et al., 2012) Therefore, regulating liver regeneration is particularly important in addition to maintaining adequate liver function in recipients of partial transplants. Furthermore, the relationship between cytokine expression and liver regeneration after cold ischemia and liver transplantation has not been explored. The current study established a stable partial liver transplantation model in rats and determined the impact of cold ischemia on the regeneration of the transplanted liver and the expression of tumor necrosis factor-alpha (TNF- $\alpha$ ) and interleukin-10 (IL-10).

\section{MATERIAL AND METHODS}

\section{Experimental animals and surgical methods}

Both donor and recipient animals were male Wistar rats provided by the Third Military Medical University (Chongqing, China) with body weights of approximately 250-300 g. The rats were fasted before surgery but provided with water, and surgery was performed sterilely under a microscope. 


\section{Donor surgery}

After rats were placed under anesthesia with ketamine, a cross incision was made in the abdomen, and the liver was partially freed. A self-made epidural catheter was inserted into the opened distal bile duct and fixed. The pyloric and splenic veins were ligated, and the portal vein was freed. The right adrenal and right renal veins were then ligated, and the left phrenic vein was ligated after the suprahepatic vena cava was freed. After the hepatic artery was separated, ligated, and cut, the left lateral lobe and part of the middle and caudate lobes (constituting approximately $50 \%$ of the liver) were removed using an in vivo liver resection method. Intravenous catheterization was performed through the superior mesenteric vein for liver perfusion using $20 \mathrm{~mL} 4^{\circ} \mathrm{C}$ saline containing $10 \mathrm{U} / \mathrm{mL}$ heparin. The donor liver was pruned, and portal vein and liver inferior vena cava cannulas were inserted.

\section{Recipient surgery}

The rat partial liver transplantation model was established on the basis of previous reports, with some modifications (Olschewski et al., 2008; Wan et al., 2008). The recipient was anesthetized with ketamine, and a midline incision was made in the abdomen. The liver was partially freed, and the hepatic artery was ligated and cut. The bile duct near the liver was cut. The portal vein segment near the liver was freed, and the right adrenal vein was ligated. After clamping the portal vein, $2 \mathrm{~mL}$ normal saline was injected at the portal vein bifurcation using a syringe. After the suprahepatic vena cava was clamped using a Satinsky clamp, the liver was removed, and the donor liver was implanted. Then, the suprahepatic vena cava was sutured using a continuous suture technique with 7-0 sutures, both the portal vein and inferior vein cannulas were ligated and fixed, and hepatic blood flow was restored.

\section{Experimental groups}

Experimental animals were randomly divided into 3 groups. The control group $(\mathrm{N}=$ 30 ) was the $50 \%$ hepatectomy group. In this group, we freed the liver from the abdominal wall and resected the left lateral lobe and part of the middle and caudate lobes to remove $50 \%$ of the liver. Experimental group $\mathrm{A}(\mathrm{N}=30)$ was the partial liver transplantation group with a cold ischemia time of less than $30 \mathrm{~min}$. After liver resection, $50 \%$ of the donor liver was subjected to cold ischemia for $30 \mathrm{~min}$ and implanted after performing the procedures described in the previous section. Experimental group $\mathrm{B}(\mathrm{N}=30)$ was the partial liver transplantation group with a cold ischemia time of $10 \mathrm{~h}$. After liver resection, $50 \%$ of the donor liver was subjected to cold ischemia for $10 \mathrm{~h}$ and implanted after the procedures described in the previous section.

\section{Immunohistochemical staining}

Staining was performed with ready-to-use PCNA and TNF- $\alpha$ staining kits, an anti-IL-10 monoclonal antibody (Genzyme Corporation, Cambridge, MA, USA), a biotin-labeled secondary immunoglobulin $\mathrm{G}$ antibody, and an avidin-biotin-peroxidase complex reagent (Vector) following manufacturer instructions. The criteria for positive immunohistochemistry results were as follows: cells were PCNA positive if the nuclei were clearly stained brown; cells were TNF- $\alpha$ 
and IL-10 positive if the cytoplasm showed obvious brown staining. The positive results were classified using the following criteria: 100 liver cells were counted per field in 6 high-power fields, and the average of the percent positive cells was calculated. The specimens were also subjected to routine hematoxylin and eosin staining, and cell morphology was observed.

\section{Statistical analysis}

Data analysis was performed using SPSS for Windows (version 13.0, SPSS Institute, Chicago, IL, USA). Differences in enumeration data were analyzed using the chi-square test. Differences in measurement data were analyzed using the $t$-test. A P value of $<0.05$ was considered to be statistically significant.

\section{RESULTS}

\section{Survival rate}

The survival rate of the $50 \%$ hepatectomy group (control group) was $100 \%$. In the experimental groups, only the animals that survived more than 3 days were counted. The animals that succumbed before 3 days were considered to have died owing to surgical complications. The group receiving a partial liver transplantation after cold ischemia for $30 \mathrm{~min}$ (experimental group A) and $10 \mathrm{~h}$ (experimental group B) had survival rates of $70 \%(21 / 30)$ and $33.3 \%$ $(10 / 30)$, respectively. A significant difference was found between these 2 groups $(\mathrm{P}<0.05)$.

\section{PCNA expression}

PCNA expression reached a peak 1 day after surgery in the control group and 2 days after surgery in both experimental groups. The peak expression of PCNA in the experimental group A was significantly higher than that in the control group, whereas the peak PCNA expression in the experimental group B was lower than that in both experimental group A and the control group (Table 1).

Table 1. Proliferating cell nuclear antigen (PCNA) expression.
\begin{tabular}{lccc}
\hline Groups & \multicolumn{3}{c}{ PCNA-positive rate (\%) } \\
\cline { 2 - 4 } & 1 day after surgery & 2 days after surgery & 4 days after surgery \\
\hline Control group & $47 \pm 3.3$ & $33 \pm 3.1$ & $24 \pm 2.8$ \\
Experimental group A & $24 \pm 3.0^{*}$ & $59 \pm 5.5$ & $38 \pm 3.4$ \\
Experimental group B & $14 \pm 2.6^{* \#}$ & $23 \pm 3.3$ & $14 \pm 3.1$ \\
\hline
\end{tabular}

Data are reported as means \pm SD for 10 animals in each group. ${ }^{*}$ Compared to the control group, $\mathrm{P}<0.05 ;{ }^{*}$ compared to the group $\mathrm{A}, \mathrm{P}<0.05$.

\section{TNF- $\alpha$ and IL-10 expression}

TNF- $\alpha$ expression reached a peak 1 day after surgery in the control group and 2 days after surgery in both experimental groups. After 2 or 4 days, the expression of TNF- $\alpha$ in the experimental group A was significantly higher than that in the control group $(\mathrm{P}<0.01)$, whereas its expression in the experimental group B was significantly lower than that in the experimental 
group A $(\mathrm{P}<0.01)$. One day after surgery, experimental groups $\mathrm{A}$ and $\mathrm{B}$ had significant higher levels of IL-10 compared with those of the control group. After 2 and 4 days, IL-10 expression was higher in the experimental group B compared with that in the experimental group $\mathrm{A}(\mathrm{P}<$ 0.05) (Table 2).

\begin{tabular}{|c|c|c|c|c|c|c|}
\hline \multirow[t]{2}{*}{ Groups } & \multicolumn{3}{|c|}{ TNF- $\alpha$-positive rate (\%) } & \multicolumn{3}{|c|}{ IL-10-positive rate (\%) } \\
\hline & 1 day after surgery & 2 days after surgery & 4 days after surgery & 1 day after surgery & 2 days after surgery & 4 days after surgery \\
\hline Control group & $60.3 \pm 3.3$ & $48.5 \pm 3.8$ & $37.4 \pm 3.2$ & $32.3 \pm 2.9$ & $38.5 \pm 3.6$ & $23.2 \pm 3.7$ \\
\hline Experimental group A & $55.3 \pm 3.7$ & $64.5 \pm 4.4$ & $61.4 \pm 3.8$ & $37.3 \pm 3.9$ & $28.5 \pm 3.3$ & $21.2 \pm 3.1$ \\
\hline Experimental group B & $48.3 \pm 3.6$ & $49.7 \pm 3.0$ & $36.4 \pm 3.6$ & $46.3 \pm 2.7$ & $36.5 \pm 3.3$ & $28.2 \pm 3.4$ \\
\hline
\end{tabular}

Data are reported as means \pm SD for 10 animals in each group.

\section{DISCUSSION}

In this study, we found that PCNA expression was increased in the 50\% hepatectomy group and peaked $24 \mathrm{~h}$ after the operation. The peak for PCNA expression was delayed in both experimental partial liver transplantation groups and was observed $48 \mathrm{~h}$ after surgery. The duration of cold ischemia significantly affected liver regeneration after transplantation. In the partial liver transplantation group with a 30-min cold ischemia time, PCNA expression was significantly higher than that of the $50 \%$ hepatectomy group 2 days after surgery $(\mathrm{P}<0.01)$, whereas the partial liver transplantation group with a 10 -h cold ischemia time had a lower PCNA expression level than that of the 50\% hepatectomy group 2 days after the operation (P $<0.05)$.

TNF- $\alpha$ is a multifunctional cytokine. In addition to being cytotoxic to tumor cells, it has an impact on liver cell function by promoting liver cell regeneration (Yang et al., 2012; Bolignano, 2012). IL-10 was first discovered in 1989 by Fiorentino et al. (1989). It inhibits the synthesis of a variety of cytokines and is, therefore, also known as a cytokine synthesisinhibitory factor. Our study showed that the 30 -min cold ischemia group had a survival rate higher than that of the 10 -h cold ischemia group $(\mathrm{P}<0.05)$, suggesting that the duration of cold ischemia impacts survival. The expression of TNF- $\alpha$ in the partial liver transplantation group with a 30 -min cold ischemia time was higher than that in the $50 \%$ hepatectomy group $(\mathrm{P}<0.05)$; however, TNF- $\alpha$ expression in the partial liver transplantation group with a 10-h cold ischemia time was lower than that in the $50 \%$ hepatectomy group $(\mathrm{P}<0.01)$. The IL-10 level in the 30-min cold ischemia group was lower than that in the 10-h cold ischemia group $(\mathrm{P}<0.05)$. These results suggest that cold ischemia time significantly affects postoperative liver regeneration and the postoperative survival rate of transplanted rats. In the process of liver regeneration, TNF- $\alpha$ and IL-10 play important roles, and a short cold ischemia period leads to increased TNF- $\alpha$ expression and decreased IL-10 expression. TNF- $\alpha$ expression correlates with the proliferative capacity of liver cells, and IL-10 may affect the capability of the liver to regenerate by downregulating TNF- $\alpha$. Our results are consistent with those of recently published studies (Klemm et al., 2008; Sudo et al., 2008; Ito et al., 2009).

The present study found that the group receiving a partial liver transplant after less than $30 \mathrm{~min}$ of cold ischemia had a proliferation peak higher than that of the $50 \%$ hepatectomy group, but the mechanism for this outcome is unclear. TNF- $\alpha$ expression was likely upregu- 
lated through some unidentified mechanism, thereby enhancing the regenerative capacity of the liver. This study thus provides some guidance for clinical liver transplantation. Studies have shown that when a living donor liver transplantation graft weighs less than $40 \%$ of the recipient liver weight, survival rate decreases significantly (Kiuchi et al., 1999; Zetzmann et al., 2010). When a sufficient amount of liver (50\% of the liver was used for transplantation in this study) is transplanted, cold ischemia time significantly affects the regenerative capacity of the liver and recipient survival rate. To some extent, our findings may explain why living donor liver transplantation is superior to split liver transplantation, as the main difference between these methods is the length of cold ischemia time. Therefore, when performing clinical split liver transplantation, cold ischemia must be shortened to the minimum effective time.

\section{REFERENCES}

Bolignano D (2012). TNF- $\alpha$ receptors (TNFRS): the biomarkers of progressive diabetic nephropathy we were waiting for? G. Ital. Nefrol. 29: 262.

Fiorentino DF, Bond MW and Mosmann TR (1989). Two types of mouse T helper cell. IV. Th2 clones secrete a factor that inhibits cytokine production by Th1 clones. J. Exp. Med. 170: 2081-2095.

Hegab B, Khalaf H, Allam N, Azzam A, et al. (2012). De novo malignancies after liver transplantation: a single-center experience. Ann. Saudi Med. 32: 355-358.

Ito H, Ando K, Ishikawa T, Saito K, et al. (2009). Role of TNF- $\alpha$ produced by nonantigen-specific cells in a fulminant hepatitis mouse model. J. Immunol. 182: 391-397.

Kele PG, de Boer M, van der Jagt EJ, Lisman T, et al. (2012). Early hepatic regeneration index and completeness of regeneration at 6 months after partial hepatectomy. Br. J. Surg. 99: 1113-1119.

Kiuchi T, Kasahara M, Uryuhara K, Inomata Y, et al. (1999). Impact of graft size mismatching on graft prognosis in liver transplantation from living donors. Transplantation 67: 321-327.

Klemm K, Eipel C, Cantre D, Abshagen K, et al. (2008). Multiple doses of erythropoietin impair liver regeneration by increasing TNF- $\alpha$, the Bax to Bcl-xL ratio and apoptotic cell death. PLoS One 3: e3924.

Li C, Mi K, Wen T, Yan L, et al. (2011). Outcomes of patients with benign liver diseases undergoing living donor versus deceased donor liver transplantation. PLoS One 6: e27366.

Lo CM (2012). Living donor liver transplantation for acute liver failure: no other choice. Liver Transpl. 18: 1005-1006.

Olschewski P, Hunold G, Eipel C, Neumann U, et al. (2008). Improved microcirculation by low-viscosity histidinetryptophan-ketoglutarate graft flush and subsequent cold storage in University of Wisconsin solution: results of an orthotopic rat liver transplantation model. Transpl. Int. 21: 1175-1180.

Omura T, Ascher NL and Emond JC (1996). Fifty-percent partial liver transplantation in the rat. Transplantation 62: 292-293.

Sudo K, Yamada Y, Saito K, Shimizu S, et al. (2008). TNF- $\alpha$ and IL-6 signals from the bone marrow derived cells are necessary for normal murine liver regeneration. Biochim. Biophys. Acta 1782: 671-679.

Wan CD, Cheng R, Wang HB and Liu T (2008). Immunomodulatory effects of mesenchymal stem cells derived from adipose tissues in a rat orthotopic liver transplantation model. Hepatobiliary Pancreat. Dis. Int. 7: 29-33.

Yang YN, Wang F, Zhou W, Wu ZQ, et al. (2012). TNF- $\alpha$ stimulates MMP-2 and MMP-9 activities in human corneal epithelial cells via the activation of FAK/ERK signaling. Ophthalmic Res. 48: 165-170.

Yu SB, Xing XH, Dong GY, Weng XL, et al. (2012). Excess genistein suppresses the synthesis of extracellular matrix in female rat mandibular condylar cartilage. Acta Pharmacol. Sin. 33: 918-923.

Zetzmann CP, Swamy OR, Loss GE Jr, Bohorquez H, et al. (2010). Improving donor livers by inhibiting TNF- $\alpha$ production. Ochsner. J. 10: 250-255. 